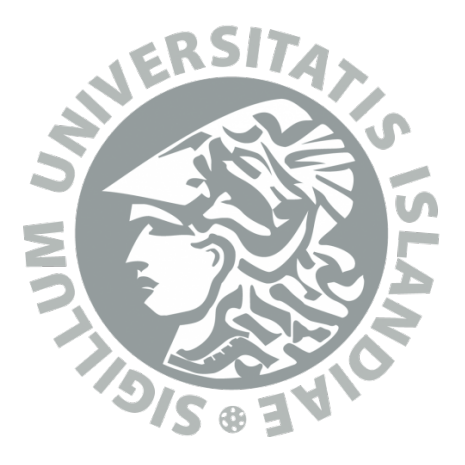

\title{
Occurrence graphs of patterns in permutations
}

\author{
Bjarni Jens Kristinsson
}

Faculty of Physical Sciences

University of Iceland

2015 



\title{
OCCURRENCE GRAPHS OF PATTERNS IN PERMUTATIONS
}

\author{
Bjarni Jens Kristinsson
}

10 ECTS thesis submitted in partial fulfillment of a Scientiæ Baccalaureus degree in Mathematics

\author{
Advisor \\ Henning Ulfarsson \\ Faculty Representative \\ Rögnvaldur Möller
}

Faculty of Physical Sciences

School of Engineering and Natural Sciences

University of Iceland

Reykjavik, June 2015 
Occurrence graphs of patterns in permutations

10 ECTS thesis submitted in partial fulfillment of a B.Sc. degree in Mathematics

Copyright (C) 2015 Bjarni Jens Kristinsson

All rights reserved

Faculty of Physical Sciences

School of Engineering and Natural Sciences

University of Iceland

Hjarðarhagi 2-6

107, Reykjavik

Iceland

Telephone: 5254000

Bibliographic information:

Bjarni Jens Kristinsson, 2015, Occurrence graphs of patterns in permutations, B.Sc. thesis, Faculty of Physical Sciences, University of Iceland.

Printing: Háskólaprent, Fálkagata 2, 107 Reykjavík

Reykjavik, Iceland, June 2015 


\section{Abstract}

This paper is based on a generalization of the idea behind the proof of the Simultaneous Shading Lemma by Claesson et al. (2014). We define the occurrence graph $G_{p}(\pi)$ of a pattern $p$ in a permutation $\pi$ as the graph with the occurrences of $p$ in $\pi$ as vertices and edges between the vertices if the occurrences differ by exactly one element. We study the general properties of the occurrence graphs and some interesting extreme cases. The main theorem in this paper is that every hereditary property of graphs produces a permutation class.

\section{Útdráttur}

Pessi grein er byggð á útvíkkun á hugmyndinni sem notuð var í sönnuninni á skyggingarhjálparsetningunni í Claesson et at. (2014). Við skilgreinum tilvikanet, $G_{p}(\pi)$, fyrir mynstur $p$ í umröðun $\pi$ sem net með hnút fyrir hvert tilvik $p$ í $\pi$ og leggi milli hnúta hvers samsvarandi tilvik eru eins fyrir utan eitt stak. Við rannsökum almenna eiginleika bessara tilvikaneta og áhugaverð jaðartilvik. Meginsetning greinarinnar er að sérhver arfgengur eiginleiki neta leiðir til umraðanaklasa. 



\section{Contents}

Acknowledgments $\quad$ ix

1 Introduction $\quad 1$

2 Permutation and graph background 3

$\begin{array}{lll}3 & \text { Occurrence graphs } & 7\end{array}$

4 The pattern $p=12$ and the identity permutation 9

$\begin{array}{lll}5 & \text { General results } & 11\end{array}$

6 Forests and trees $\quad 15$

$\begin{array}{lll}7 & \text { Future work } & 25\end{array}$

$\begin{array}{lr}\text { Bibliography } & 27\end{array}$ 



\section{Acknowledgments}

My sincerest thanks go to Henning Ulfarsson for taking me on as his student. I thank him for his support and guidance and for being a kind and patient, yet demanding, mentor. I would also like to thank my girlfriend, Kristrún Skúladóttir, for her support and proofreading and for bearing with me all this time. 



\section{Introduction}

In this article we extend an idea originating from the proof of the Simultaneous Shading Lemma made by Claesson, Tenner and Ulfarsson in [1]. Our extension is to construct a graph from the various stages in the algorithm and the steps that the algorithm takes to transform between them. The reader does not need to familiarize himself with the Simultaneous Shading Lemma as everything in this paper stands independently from the lemma. The main theorem in this paper is that every hereditary property of graphs produces a permutation class. 



\section{Permutation and graph background}

In this article we will be working with permutations and undirected, simple graphs. The reader does not need to have prior knowledge of either as we will define both. We will then define occurrence graphs made from patterns in permutations and present our research results.

Definition 2.1. A simple graph is an ordered pair $G=(V, E)$ where $V$ is a set of vertices and $E$ is a set of two-element subsets of $V$. The elements $\{u, v\} \in E$ are called edges and connect the vertices. Two vertices $u$ and $v$ are called neighbors if $\{u, v\} \in E$. The degree of a vertex $v$ is the number of neighbors it has. A graph $G^{\prime}=\left(V^{\prime}, E^{\prime}\right)$ is a subgraph in $G$ if $V^{\prime} \subseteq V$ and $E^{\prime} \subseteq\left\{\{u, v\} \in E: u, v \in V^{\prime}\right\}$.

The reader might have noticed that our definition of a graph exludes those with loops and multiple edges between vertices. Throughout the article we will only be working with simple, unweighted and undirected graphs. We often write $u v$ as shorthand for $\{u, v\}$ and in case of ambiguity we use $V(G)$ and $E(G)$ instead of $V$ and $E$.

Definition 2.2. Let $V^{\prime}$ be a subset of $V$. The induced subgraph $G\left[V^{\prime}\right]$ is a subgraph of $G$ with vertex set $V^{\prime}$ and edges $\left\{u v \in E: u, v \in V^{\prime}\right\}$.

Two graphs $G$ and $H$ are isomorphic if there exist a bijection from $V(G)$ to $V(H)$ such that two vertices in $G$ are neighbors if and only if the corresponding vertices (according to the bijection) in $H$ are neighbors. We denote this with $G \cong H$.

We let $\llbracket 1, n \rrbracket$ denote the integer interval $\{1, \ldots, n\}$.

Definition 2.3. A permutation of length $n$ is a bijective function $\sigma: \llbracket 1, n \rrbracket \rightarrow \llbracket 1, n \rrbracket$. We denote the permutation with $\sigma=\sigma(1) \sigma(2) \cdots \sigma(n)$. The permutation $\operatorname{id}_{n}=$ $12 \cdots n$ is the identity permutation of length $n$.

A permutation is simply a description of what to do with an enumerated list of $n$ entries. Computer scientists and programmers often work with data structures called 
lists and vectors where the elements are enumerated from 1 to $n$. A permutation on the list would result in a new or modified list where the elements have been rearranged according to the permutation. For example, the permutation $\sigma=35142$ sorts the list $a=[12,28,9,27,11]$ because $a(3)=9<a(5)=11<a(1)=12<$ $a(4)=27<a(2)=28$.

The set of permutations of length $n$ is denoted by $\mathfrak{S}_{n}$. The set of all permutations is $\mathfrak{S}=\cup_{n=0}^{+\infty} \mathfrak{S}_{n}$. Note that $\mathfrak{S}_{0}=\{\mathscr{E}\}$, where $\mathscr{E}$ is the empty permutation, and $\mathfrak{S}_{1}=\{1\}$. There are $n$ ! permutations of length $n$.

Definition 2.4. A grid plot or grid representation of a permutation $\pi \in \mathfrak{S}_{n}$ is the subset $\operatorname{Grid}(\pi)=\{(i, \pi(i)): i \in \llbracket 1, n \rrbracket\}$ of the Cartesian product $\llbracket 1, n \rrbracket^{2}=$ $\llbracket 1, n \rrbracket \times \llbracket 1, n \rrbracket$.

Example 2.5. Let $\pi=42135$. The grid representation of $\pi$ is

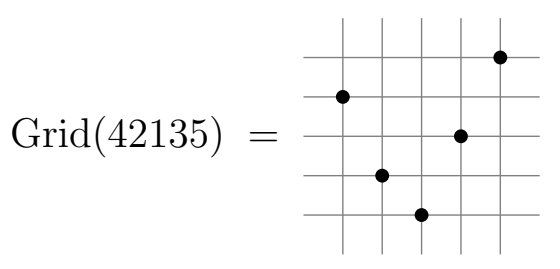

Figure 2.1: The grid plot of the permutation 42135

One of the central definitions of this paper is how permutations can lie inside other (larger) permutations. Before we define that precisely we need a preliminary definition:

Definition 2.6. Let $a_{1}, \ldots, a_{k}$ be distinct integers. The standardisation of the string $a_{1} \cdots a_{k}$ is the permutation $\sigma \in \mathfrak{S}_{k}$ such that $a_{1} \cdots a_{k}$ is order isomorphic to $\sigma(1) \cdots \sigma(k)$. In other words, for every $i \neq j$ we have $a_{i}<a_{j}$ if and only if $\sigma(i)<\sigma(j)$. We denote this with $\operatorname{st}\left(a_{1} \cdots a_{k}\right)=\sigma$.

For example $\operatorname{st}(253)=132$ and $\operatorname{st}(132)=132$.

Definition 2.7. Let $p$ be a permutation of length $k$. We say that the permutation $\pi \in \mathfrak{S}_{n}$ contains $p$ if there exist indices $1 \leq i_{1}<\cdots<i_{k} \leq n$ such that st $\left(\pi\left(i_{1}\right) \cdots \pi\left(i_{k}\right)\right)=p$. The subsequence $\pi\left(i_{1}\right) \cdots \pi\left(i_{k}\right)$ is an occurrence of $p$ in $\pi$ with the index set $\left\{i_{1}, \ldots, i_{k}\right\}$. The increasing sequence $i_{1} \cdots i_{k}$ is a denotement for the order preserving injection $i: \llbracket 1, k \rrbracket \rightarrow \llbracket 1, n \rrbracket, j \mapsto i_{j}$ which we call the index injection of $p$ into $\pi$ for this particular occurrence.

The set of all index sets of $p$ in $\pi$ is the occurrence set of $p$ in $\pi$, denoted with $V_{p}(\pi)$. If $\pi$ does not contain $p$, then $\pi$ avoids $p$. In this context the permutation $p$ is called a (classical permutation) pattern. 
Unless otherwise stated, we write the index set $\left\{i_{1}, \ldots, i_{n}\right\}$ in ordered form, i.e. such that $i_{1}<\cdots<i_{n}$, in accordance with how we write the index injection.

The set of all permutations that avoid $p$ is $\operatorname{Av}(p)$. More generally for a set of patterns $M$ we define

$$
\operatorname{Av}(M)=\bigcap_{p \in M} \operatorname{Av}(p)
$$

Example 2.8. The permutation 42135 contains five occurrences of the pattern 213, namely 425, 415, 435, 213 and 215 . The occurrence set is

$$
V_{213}(42135)=\{\{1,2,5\},\{1,3,5\},\{1,4,5\},\{2,3,4\},\{2,3,5\}\} .
$$

The permutation 42135 avoids the pattern 132 . 



\section{Occurrence graphs}

Definition 3.1. For a pattern $p \in \mathfrak{S}_{k}$ and for a permutation $\pi \in \mathfrak{S}_{n}$ we define the occurrence graph $G_{p}(\pi)$ of $p$ in $\pi$ in the following way:

- The set of vertices is $V_{p}(\pi)$, the occurrence set of $p$ in $\pi$.

- $u v$ is an edge in $G_{p}(\pi)$ if the vertices $u=\left\{u_{1}, \ldots, u_{k}\right\}$ and $v=\left\{v_{1}, \ldots, v_{k}\right\}$ in $V_{p}(\pi)$ differ by exactly one element, i.e. if

$$
|u \backslash v|=|v \backslash u|=1
$$

Example 3.2. In Example 2.8 we derived the occurrence set $V_{213}(42135)$. We compute the edges of $G_{213}(42135)$ by comparing the vertices two at a time to see if the sets differ by exactly one element. The graph is shown in Figure 3.1.

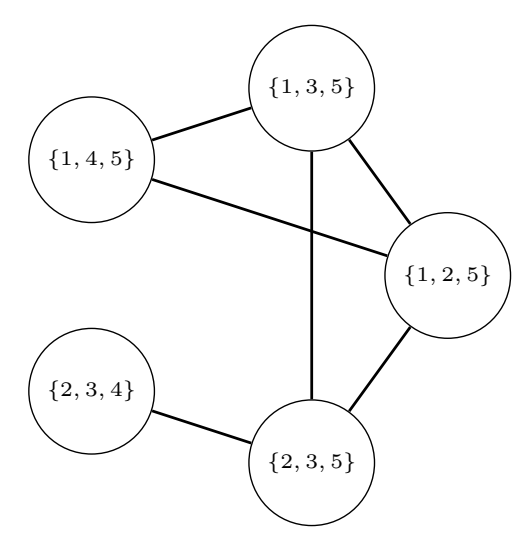

Figure 3.1: The occurrence graph $G_{213}(42135)$

Remark 3.3. For $\pi \in \mathfrak{S}_{n}$ we note that $G_{\mathscr{E}}(\pi)$ is a graph with one vertex and no edges and $G_{1}(\pi)$ is a clique on $n$ vertices.

Following the definition of these graphs there are several natural questions that arise. For example, how many occurrence graphs satisfy a given property such as being connected or being a tree? 



\section{The pattern $p=12$ and the identity permutation}

Let's fix $p=12$ and let $n \geq 2$. For this choice of $p$ and a fixed $n$ the identity permutation $\pi=1 \cdots n$ contains the most occurrences of $p$. This extreme case makes for an interesting occurrence graph $G=G_{p}(\pi)$.

Every set $\{i, j\}$ with $i \neq j$ is an index set of $p$ in $\pi$. We can choose this pair in

$$
\left(\begin{array}{l}
n \\
2
\end{array}\right)=\frac{n(n-1)}{2}
$$

different ways. Therefore, this is the size of the vertex set $V(G)=V_{p}(\pi)$.

Every vertex $u=\{i, j\}$ in $G$ is connected to $n-2$ vertices $v=\left\{i, j^{\prime}\right\}, j^{\prime} \neq j$, and $n-2$ vertices $w=\left\{i^{\prime}, j\right\}, i^{\prime} \neq i$. Thus, the degree of every vertex in $G$ is $2(n-2)$. By summing this over the set of vertices and dividing by two we get the number of edges in $G$ :

$$
|E(G)|=\frac{n(n-1)(n-2)}{2}=3\left(\begin{array}{l}
n \\
3
\end{array}\right)
$$

For every number $i \in \llbracket 1, n \rrbracket$ the induced subgraph of $\{\{i, j\}: j \neq i\}$ in $G$ is isomorphic to $K_{n-1}$. There are $n$ such subgraphs in $G$.

A triangle in $G$ are three vertices $u, v, w$ with edges $u v, v w, w u$. If $u=\{i, j\}$ (not neccessarily in ordered form) then $v$ must be of the form $\{j, k\}$, because $u$ and $v$ have exactly one element in common. For this triplet to be a triangle $w$ must connect to both $u$ and $v$, and therefore $w$ must either be the index set $\{i, k\}$ or of the form $\left\{j, j^{\prime}\right\}$ where $j^{\prime} \neq i, k$.

In the latter case the triangle lies inside a subgraph that is isomorphic to $K_{n-1}$. For each of the $n$ such subgraphs, we can choose any three vertices to make up a triangle, and therefore there are $n\left(\begin{array}{c}n-1 \\ 3\end{array}\right)$ such triangles in $G$.

In the former case, we can regard the three vertices $u=\{i, j\}, v=\{j, k\}$ and $w=\{i, k\}$ as coming from seperate $K_{n-1}$ subgraphs, induced by $i, j$ and $k$. We can 
choose the three numbers $i, j, k$, and therefore the three subgraphs, in $\left(\begin{array}{l}n \\ 3\end{array}\right)$ different ways.

By summing these two cases together we get that the number of triangles in $G$ is

$$
n\left(\begin{array}{c}
n-1 \\
3
\end{array}\right)+\left(\begin{array}{l}
n \\
3
\end{array}\right)=(n-2)\left(\begin{array}{l}
n \\
3
\end{array}\right) .
$$

Example 4.1. The graph $G_{12}(12345)$ is pictured in Figure 4.1. Notice the five subgraphs that are isomorphic to $K_{4}$, one of them highlighted with bolder gray edges and gray vertices.

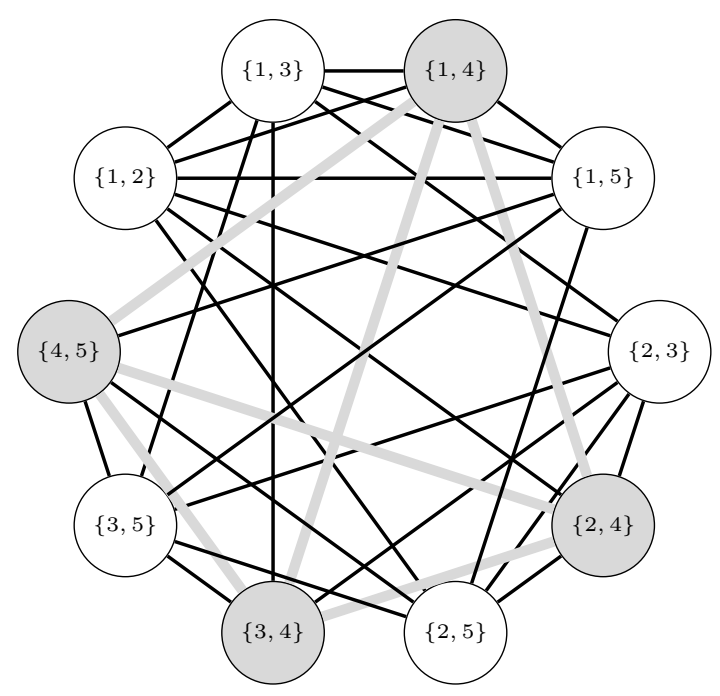

Figure 4.1: The graph $G_{12}(12345)$

In Example 4.1, the induced subgraph of $\{\{1,4\},\{2,4\},\{3,4\},\{4,5\}\}$ is a clique in $G_{12}(12345)$ because it is isomorphic to the complete graph $K_{4}$.

These examples lead us to wonder more generally about cliques in occurrence graphs with fixed pattern $p=12$ and identity permutations $\pi=\mathrm{id}_{n}$. For a fixed $n$, how many cliques are there of size $k$ in $G_{p}(\pi)$ ?

We were not able to prove it, but computer calculations with the open-source mathematical software Sage [4] indicate that $k=1$ and $k=3$ are odd cases and the usual case follows the same formula as $k=2$.

Conjecture 4.2. For $n>0$, the number of cliques of size $k>3$ in $G_{12}\left(\mathrm{id}_{n}\right)$ is

$$
(k+1)\left(\begin{array}{c}
n \\
k+1
\end{array}\right)=n\left(\begin{array}{c}
n-1 \\
k
\end{array}\right) .
$$




\section{General results}

Intuitively one would think that if a pattern $p$ is contained inside a larger pattern $q$, that either of the occurrence graphs $G_{p}(\pi)$ and $G_{q}(\pi)$ (for any permutation $\pi$ ) would be contained inside the other. But this is not the case as the following examples demonstrate.

Example 5.1. 1. Let $p=12, q=231$ and $\pi=3421$. The occurrence sets are $V_{p}(\pi)=\{\{1,2\}\}$ and $V_{q}(\pi)=\{\{1,2,3\},\{1,2,4\}\}$. The cardinality of the set $V_{p}(\pi)$ is smaller then the cardinality of $V_{q}(\pi)$.

2. If on the other hand $p=12, q=123$ and $\pi=123$ then the occurrence sets are $V_{p}(\pi)=\{\{1,2\},\{1,3\},\{2,3\}\}$ and $V_{q}(\pi)=\{\{1,2,3\}\}$. The relative size of the occurrence sets are now changed.

However, we can argue some kind of containment of one occurrence graph inside the other if we fix the pattern $p$ and let one permutation contain the other.

Lemma 5.2. Let $\pi$ and $\sigma$ be two permutations. For every occurrence of $\pi$ in $\sigma$ the index injection induces an injection $\Phi_{p}: V_{p}(\pi) \rightarrow V_{p}(\sigma)$, for all patterns $p$.

Proof. Let $p, \pi, \sigma$ be permutations of length $l, m, n$ respectively. Every $v=$ $\left\{i_{1}, \ldots, i_{l}\right\} \in V_{p}(\pi)$ is an index set of $p$ in $\pi$ with index injection $i$. Let $j$ be an index injection for an index set $\left\{j_{1}, \ldots, j_{m}\right\}$ of $\pi$ in $\sigma$. It's easy to see that $u=\left\{j_{i_{1}}, \ldots, j_{i_{l}}\right\}$ is an index set of $p$ in $\sigma$ because $j \circ i$ is an index injection of $p$ into $\sigma$. Define $\Phi_{p}(v)=u$.

Example 5.3. Let $p=12, \pi=132$ and $\sigma=24153$. There are three occurrences of $\pi$ in $\sigma: 243,253$ and 153 with respective index injections 125, 145 and 345.

For a given index injection, say $i=125$, we obtain the injection $\Phi_{p}$ by mapping every $\left\{v_{1}, v_{2}\right\} \in V_{p}(\pi)$ to $\left\{i_{v_{1}}, i_{v_{2}}\right\} \in V_{p}(\sigma)$. As there are only two occurrences of $p$ in $\pi$ we can quickly calculate that $\{1,2\} \in V_{p}(\pi)$ maps to $\left\{i_{1}, i_{2}\right\}=\{1,2\} \in V_{p}(\sigma)$ and $\{1,3\}$ maps to $\left\{i_{1}, i_{3}\right\}=\{1,5\}$.

For the index injection $i^{\prime}=345$ we calculate that $\Phi_{p}$ maps $\{1,2\}$ to $\left\{i_{1}^{\prime}, i_{2}^{\prime}\right\}=\{3,4\}$ and $\{1,3\}$ to $\left\{i_{1}^{\prime}, i_{3}^{\prime}\right\}=\{3,5\}$, see Figure 5.1. 


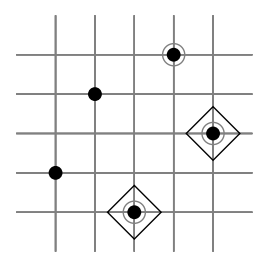

Figure 5.1: The occurrence of $\pi$ in $\sigma$ that is defined by the index injection $i^{\prime}=345$ is highlighted with gray circles. The occurrence set $\{1,3\}$ of $p$ in $\pi$ is mapped with the injection $\Phi_{p}$, induced by $i^{\prime}$, to the occurrence set $\{3,5\}$ of $p$ in $\sigma$, highlighted with black diamonds

Lemma 5.4. Let $\pi$ and $\sigma$ be two permutations. For every occurrence of $\pi$ in $\sigma$ the index injection induces an isomorphism of the occurrence graph $G_{p}(\pi)$ with a subgraph in $G_{p}(\sigma)$, for all patterns $p$.

Proof. From Lemma 5.2 we have the injection $\Phi_{p}: V_{p}(\pi) \rightarrow V_{p}(\sigma)$. We need to show that for every $u v \in E\left(G_{p}(\pi)\right)$ that $\Phi_{p}(u) \Phi_{p}(v) \in E\left(G_{p}(\sigma)\right)$.

To complete the proof let $u v$ be an edge in $G_{p}(\pi)$, where $u=\left\{u_{1}, \ldots, u_{l}\right\}$ and $v=\left\{v_{1}, \ldots, v_{l}\right\}$. For every index injection $j$ of $\pi$ into $\sigma$, the vertices $u, v$ map to $\Phi_{p}(u)=\left\{j\left(u_{1}\right), \ldots, j\left(u_{l}\right)\right\}, \Phi_{p}(v)=\left\{j\left(v_{1}\right), \ldots, j\left(v_{l}\right)\right\}$ respectively. Since $j$ is an injection there exist an edge between these two vertices in $G_{p}(\sigma)$.

Example 5.5. We will continue with Example 5.3 and show how the index injections $i=125$ and $i^{\prime}=345$ define subgraphs of $G_{p}(\sigma)$ which are isomorphic to $G_{p}(\pi)$. The occurrence graph of $p$ in $\pi$ is a graph on two vertices $\{1,2\}$ and $\{1,3\}$ with an edge between them. The occurrence graph $G_{p}(\sigma)$ with the highlighted subgraphs induced by $i$ and $i^{\prime}$ that are isomorphic to $G_{p}(\pi)$ are shown in Figure 5.2.

The next example shows that different occurrences of $\pi$ in $\sigma$ do not necessarily lead to different subgraphs in $G_{p}(\sigma)$.

If $p=12, \pi=312$ and $\sigma=3412$ there are two occurrences of $\pi$ in $\sigma$. The index injections are $i=134$ and $i^{\prime}=234$. However, as $\left(i_{2}, i_{3}\right)=\left(i_{2}^{\prime}, i_{3}^{\prime}\right)$ and the fact that $\{1,2\}$ is the only occurrence set of $p$ in $\pi$, we obtain the same injection $\Phi_{p}$ and therefore the same subgraph in $G_{p}(\sigma)$ for both index injections.

We call a property of a graph $G$ hereditary if it is invariant under isomorphisms and for every subgraph of $G$ the property also holds. For example the properties of being a forest, bipartite, planar or $k$-colorable are hereditary properties. The property of being a tree is not hereditary. 

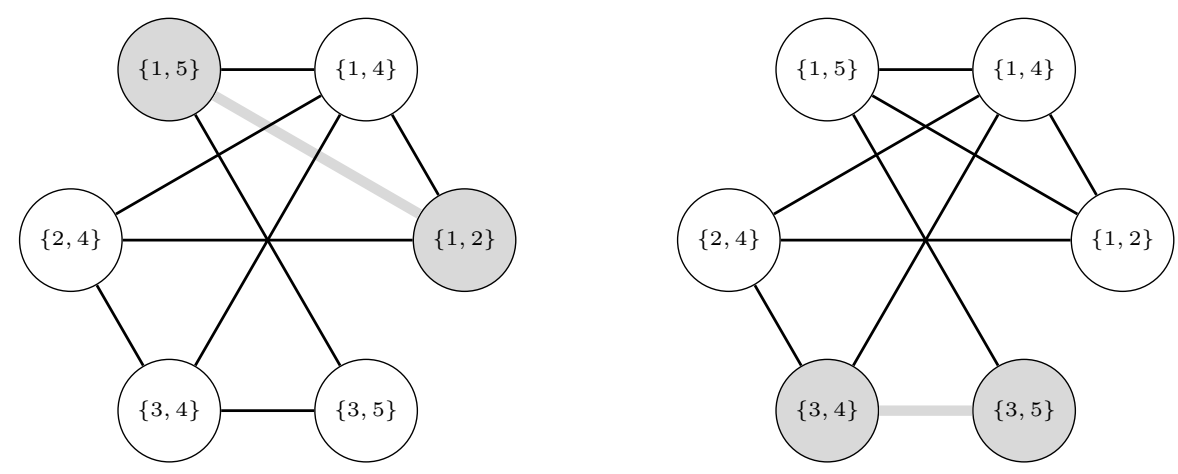

Figure 5.2: The graph $G_{12}(24153)$ with highlighted subgraphs that are isomorphic to $G_{12}(132)$. On the left the subgraph is induced by the index injection $i$ and on the right by $i^{\prime}$

Let $c$ be a property of graphs and let

$$
\mathscr{G}_{p, c}=\left\{\pi \in \mathfrak{S}: G_{p}(\pi) \text { has property } c\right\} .
$$

Theorem 5.6. Let $c$ be a hereditary property of graphs. For any pattern $p$ the set $\mathscr{G}_{p, c}$ is a permutation class, i.e. there is a set of classical permutations patterns $M$ such that

$$
\mathscr{G}_{p, c}=\operatorname{Av}(M)
$$

Proof. Let $\sigma$ be a permutation such that $G_{p}(\sigma)$ satisfies the hereditary property $c$ and let $\pi$ be a pattern in $\sigma$. By Lemma 5.4 the graph $G_{p}(\pi)$ is isomorphic to a subgraph in $G_{p}(\sigma)$ and thus inherits the property $c$.

We will now focus on the pattern $p=12$. 



\section{Forests and trees}

Recall that a non-empty simple graph on $n$ vertices $(n>0)$ is a tree if and only if it is connected and has $n-1$ edges. An equivalent condition is that the graph has at least one vertex and no simple cycles (a simple cycle is a sequence of unique vertices $v_{1}, \ldots, v_{k}$ with edges $\left.v_{1} v_{2}, \ldots, v_{k-1} v_{k}, v_{k} v_{1}\right)$. A forest is a distinct union of trees. The empty graph is a forest but not a tree. If a graph does not contain any cycle it is acyclic.

Theorem 6.1. Let $c$ be the property of being a forest and $p=12$. Then

$$
\mathscr{G}_{p, c}=\operatorname{Av}(123,1432,2143,3214) .
$$

Before we can prove this theorem we need a proposition about the length of cycles in occurrence graphs $G_{12}(\pi)$.

Proposition 6.2. If $G_{12}(\pi)$ has a cycle then it has a cycle of length 3 or 4.

Proof. Let $p=12$ and let $\pi$ be a permutation such that $G_{p}(\pi)$ contains a cycle of length $k$. Name the vertices in the cycle $v_{1}, \ldots, v_{k}$ with $v_{l}=\left\{i_{l}, j_{l}\right\}, i_{l}<j_{l}$, for $l=1, \ldots, k$ and note that there are edges between adjacent vertices in the cycle.

The vertices $v_{1}$ and $v_{2}$ in the cycle have exactly one index in common, say $i_{1}=i_{2}$. If $j_{1}<j_{2}$ and $\pi\left(j_{1}\right)<\pi\left(j_{2}\right)$ (or $j_{1}>j_{2}$ and $\left.\pi\left(j_{1}\right)>\pi\left(j_{2}\right)\right)$ then $u=\left\{j_{1}, j_{2}\right\}$ is an occurrence of $p$ in $\pi$, forming a triangle $v_{1}, v_{2}, u$ in $G_{p}(\pi)$. So either $j_{1}>j_{2}$ and $\pi\left(j_{1}\right)<\pi\left(j_{2}\right)$ holds, or, as pictured in Figure $6.1, j_{1}<j_{2}$ and $\pi\left(j_{1}\right)>\pi\left(j_{2}\right)$.

Next we look at the edge $v_{2} v_{3}$ in the cycle. If the vertices have the index $i_{1}$ in common then $v_{1}, v_{2}, v_{3}$ forms a triangle in $G_{p}(\pi)$. So assume that $v_{2}$ and $v_{3}$ have the index $j_{2}$ in common with the conditions $i_{3}>j_{1}$ and $\pi\left(i_{3}\right)<\pi\left(i_{1}\right)$ (because else there are more vertices and edges forming a cycle of length $\leq 4$ in $G_{p}(\pi)$ ). Continuing down this road we know that $v_{3} v_{4}$ is an edge with shared index $i_{3}$ and conditions $j_{3}>i_{3}$ and $\pi\left(j_{3}\right)<\pi\left(i_{1}\right)$, see Figure 6.2.

Graphicly, it is quite obvious that we cannot extend the graphical line path in Figure 6.2 with more southwest-northeast line segments (a sequence of vertices 
6 Forests and trees

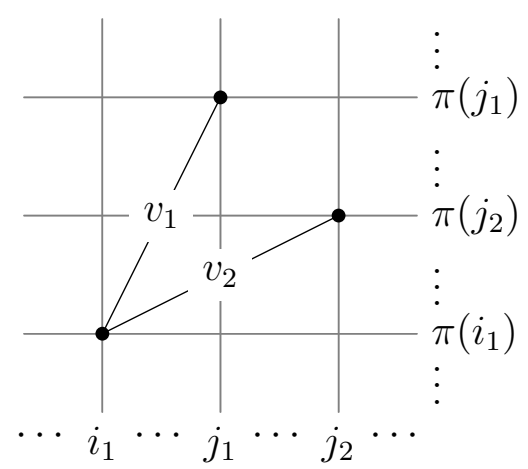

Figure 6.1: The vertices $v_{1}$ and $v_{2}$ (shown as line segments inside the permutation $\pi)$ share the index $i_{1}$. We picture the case where $j_{1}<j_{2}$ and $\pi\left(j_{1}\right)>\pi\left(j_{2}\right)$

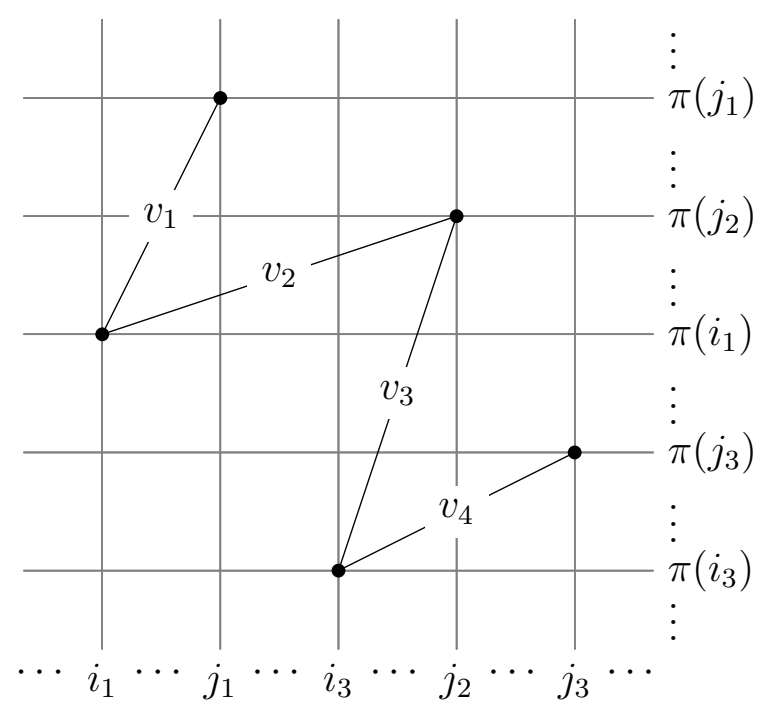

Figure 6.2: The vertices $v_{1}, v_{2}, v_{3}, v_{4}$ are shown as line segments with order relative conditions on the indices $i_{1}, i_{3}, j_{1}, j_{2}, j_{3}$ and their values inside $\pi$ 
$\left.v_{5}, \ldots, v_{k}\right)$ such that the extension closes the path into a cycle without adding more edges (line segments) between vertices that are not adjacent in the cycle and thus forming a cycle of length 3 or 4 in the occurrence graph.

For every pair of dots in the grid representation of $\pi$, because $p=12$, there is a line segment (a vertex in the occurrence graph) between them if the dot on the right lies above the dot on the left (if the line segment between the dots lies southwestnortheast).

Because we cannot close this path into a cycle, $k$ cannot be $\geq 5$, meaning that if there exist a cycle in the occurrence graph, there exist a cycle of length 3 or 4 in the graph. A similar graphical approach is used for the other cases.

Proof of Theorem 6.1. If $\pi$ contains the pattern 2143 then $G_{p}(\pi)$ contains a subgraph that is ismorphic to a cycle of length four, according to Lemma 5.4, because $G_{p}(2143)$ is a cycle of length four. If $\pi$ contains any of the patterns 123 , 1432,3214 then $G_{p}(\pi)$ contains a subgraph that is isomorphic to a triangle. So if $\pi \notin \operatorname{Av}(123,1432,2143,3214)$ then $G_{p}(\pi)$ contains a cycle and is therefore not a forest.

On the other hand, let $\pi$ be a permutation such that $G_{p}(\pi)$ is not a forest. Then the occurrence graph contains a cycle and in particular a shortest cycle. From Proposition 6.2 we know that the length of the shortest cycle in $G_{p}(\pi)$ is either 3 or 4. But it's easy to see that the only permutations whose occurrence graph is a cycle of length 3 or 4 are 123,1432,2143,3214. Therefore $\pi$ must contain at least one of the patterns.

The rest of this section is devoted to counting occurrence graphs that have the property of being a tree, which we note is not a hereditary property. We start by introducing a new notation.

Definition 6.3. Let $\pi \in \mathfrak{S}_{n}$ and $k$ be an integer such that $1 \leq k \leq n+1$. The $k$-prefix of $\pi$ is the permutation $\pi^{\prime} \in \mathfrak{S}_{n+1}$ defined by $\pi^{\prime}(1)=k$ and

$$
\pi^{\prime}(i+1)= \begin{cases}\pi(i) & \text { if } \pi(i)<k \\ \pi(i)+1 & \text { if } \pi(i) \geq k\end{cases}
$$

for $i=1, \ldots, n$. We denote $\pi^{\prime}$ with $k \succ \pi$. In a similar way we define the $k$-postfix of $\pi$ as the permutation $\pi \prec k$ in $\mathfrak{S}_{n+1}$.

This notation is chosen because of how graphical it is. Other notations have been introduced such as an up-arrow in Vidarsdottir and Ulfarsson [3]. 
Example 6.4. Let $\pi=42135$ and $k=2$. Visually, if we draw the grid representation of $\pi$, we are putting the new number $k$ to the left on the $x$-axis and raising all the numbers $\geq k$ on the $y$-axis by one. Thus we have $2 \succ 42135=253146$.

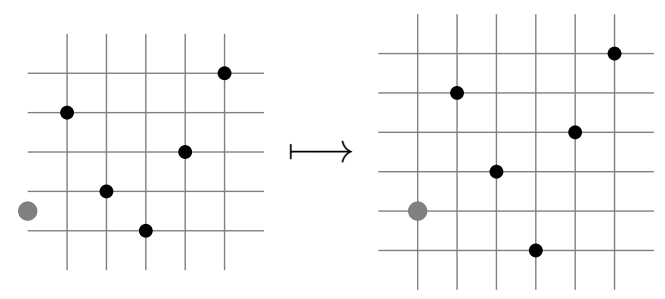

Figure 6.3: The 2-prefix of 42135 is 253146

We note that for every permutation $\pi^{\prime} \in \mathfrak{S}_{n+1}$ there is one and only one pair $(k, \pi)$ such that $\pi^{\prime}=k \succ \pi$. We let $k=\pi^{\prime}(1)$ and $\pi=\operatorname{st}\left(\pi^{\prime}(2) \cdots \pi^{\prime}(n+1)\right)$.

Example 6.5. For every decreasing permutation $\pi=n \cdots 1$ the occurrence graph of 12 in the $(n+1)$-postfix $\pi \prec(n+1)$ is a fully connected graph on $n$ vertices and therefore isomorphic to $K_{n}$. Every two vertices from the occurrence set $\{\{i, n+$ $1\}: i=1, \ldots, n\}$ share exactly one element with each other, see Figure 6.4. The same is true for the 1 -prefix, i.e. $G_{12}(1 \succ \pi) \cong G_{12}(\pi \prec(n+1)) \cong K_{n}$.
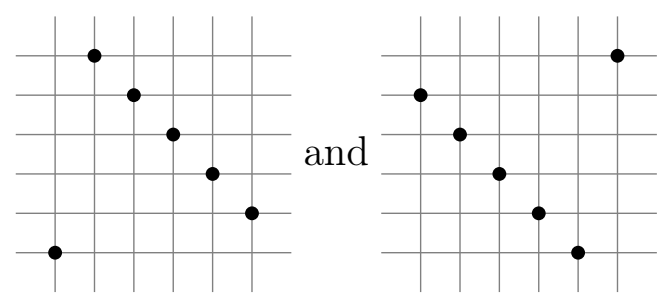

Figure 6.4: The Grid plots of $1 \succ 54321$ and $54321 \prec 6$

One of our very first discoveries was a nice sequence of quadratic numbers. We discovered this with the help of Sage [4] and proved it with induction.

Theorem 6.6. The number of permutations of length $n$ in $\mathscr{G}_{12, \text { tree }}$ is $(n-1)^{2}$.

Proof. Let $p=12$. We start by considering three base-cases.

For $n=1$ the occurrence graph is the empty graph. For $n=2$ we get two occurrence graphs: $G_{p}(12)$ is a single node graph and $G_{p}(21)$ is the empty graph. For $n=3$ we have $3 !=6$ different permutations $\pi$. Of those we calculate that $132,213,231$ 
and 312 result in connected occurrence graphs on one or two nodes but $G_{p}(123)$ is a triangle and $G_{p}(321)$ is the empty graph.

We have thus showed that the claimed enumeration is true for $n=1,2,3$.

For the inductive step we assume $n \geq 4$ and let $\pi$ be a permutation of length $n$. We look at four different cases of $k$ to construct $\pi^{\prime}=k \succ \pi$. We let $x, y$ and $z$ be the indices of $n-1, n$ and $n+1$ in $\pi^{\prime}$ respectively.

(I) $k \leq n-2$ : The index sets $\{1, x\},\{1, y\}$ and $\{1, z\}$ of $p$ in $\pi^{\prime}$ all share exactly one common element and thus form a triangle in $G_{p}\left(\pi^{\prime}\right)$. Therefore there are no permutations $\pi$ resulting in the occurrence graph $G_{p}\left(\pi^{\prime}\right)$ being a tree.

(II) $k=n-1$ : Let $T(n+1)$ denote the number of permutations $\pi^{\prime}$ of length $n+1$ with $\pi^{\prime}(1)=n-1$ such that $G_{p}\left(\pi^{\prime}\right)$ is a tree. Note that $T(1)=T(2)=0$, $T(3)=1$ and $T(4)=2$. In order to obtain a formula for $T$ we need to look at a few subcases:

i) If $y<z$ then $\{1, y\},\{1, z\}$ and $\{y, z\}$ form an triangle in $G_{p}\left(\pi^{\prime}\right)$, see Figure 6.5. Independent of the permutation $\pi$, the graph $G_{p}\left(\pi^{\prime}\right)$ is not a tree.

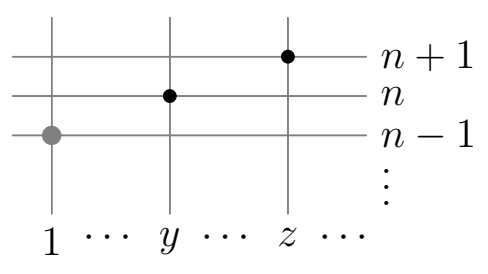

Figure 6.5: $k=n-1$ and $y<z$

ii) Assume $y>z$ and $z \neq 2$, see Figure 6.6. Then $\pi^{\prime}(2)<n-1$ and $\{1, z\}$, $\{2, z\},\{2, y\}$ and $\{1, y\}$ form a cycle of length 4 in $G_{p}\left(\pi^{\prime}\right)$, resulting in it not being a tree.

iii) Now lets assume $y>z$ and $z=2$, see Figure 6.7.

If $y \geq 5$ then the vertices $\{1, y\},\{3, y\}$ and $\{4, y\}$ form a cycle in $G_{p}\left(\pi^{\prime}\right)$.

If $y=3$ then $\{1,2\}$ and $\{1,3\}$ will be an isolated path component in $G_{p}\left(\pi^{\prime}\right)$, making $\pi^{\prime}=(n-1)(n+1) n(n-2) \cdots 1$ the only permutation such that the occurrence graph $G_{p}\left(\pi^{\prime}\right)$ is a tree.

Now fix $y=4$ and lets look at some subsubcases for the value of $\pi^{\prime}(3)$. 


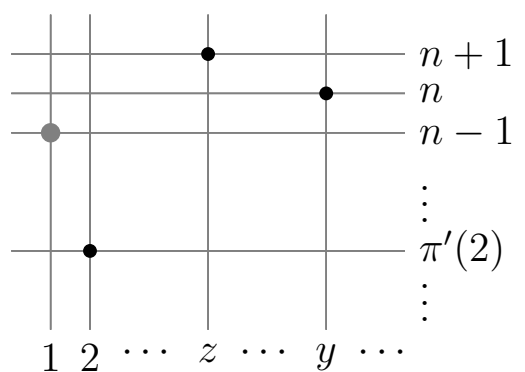

Figure 6.6: $k=n-1, y>z$ and $z \neq 2$

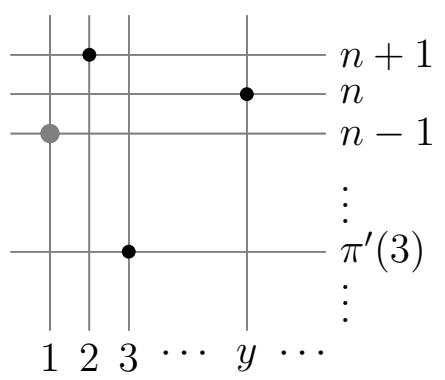

Figure 6.7: $k=n-1, y>z$ and $z=2$ 
a) If $\pi^{\prime}(3) \leq n-4$ then $\pi^{\prime}(3) n, \pi^{\prime}(3)(n-2)$ and $\pi^{\prime}(3)(n-3)$ are all occurrences of $p$ in $\pi^{\prime}$, with the respective index sets forming an triangle in $G_{p}\left(\pi^{\prime}\right)$.

b) If $\pi^{\prime}(3)=n-2$ then $\pi^{\prime}=(n-1)(n+1)(n-2) n(n-3) \cdots 1$ is the only permutation resulting in $G_{p}\left(\pi^{\prime}\right)$ being a tree.

c) If $\pi^{\prime}(3)=n-3$ we look at Figure 6.8 where the permutation $\pi^{\prime}$ is shown.

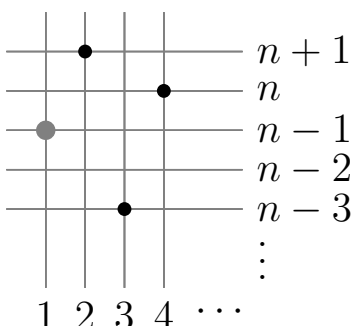

Figure 6.8: $k=n-1, y=4$ and $z=2$

The permutation $\sigma=\operatorname{st}\left(\pi^{\prime}(3) \cdots \pi^{\prime}(n+1)\right)$ is just like $\pi^{\prime}$ in the case $k=n-1$ and $z=2$, only the length of $\sigma$ is $n-1$. Because $\{1,2\}$ is a vertex in $G_{p}(\sigma)$ the occurrence graph of $p$ in $\sigma$ is not the empty graph. Thus it is easy to see that $G_{p}\left(\pi^{\prime}\right)$ is a tree if and only if $G_{p}(\sigma)$ is a tree, and according to the aforementioned case there are $T(n-1)$ such permutations $\sigma$.

Summing up the subsubcases there are a total of $1+1+T(n-1)$ permutations $\pi^{\prime}$ making the occurrence graph a tree, i.e. $T(n+1)=2+T(n-1)$. Because $T(4)=2$ and $T(3)=1$ we deduce that $T(n+1)=n-1$.

The whole case $k=n-1$ gives us that there are $n-1$ permutation $\pi^{\prime}$ such that $G_{p}\left(\pi^{\prime}\right)$ is a tree.

(III) $\underline{k=n}$ : We need to examine three subcases:

i) If $z \geq 4$ then $\{1, z\},\{2, z\},\{3, z\}$ are all index sets of $p$ in $\pi^{\prime}$, forming a triangle in $G_{p}\left(\pi^{\prime}\right)$.

ii) If $z=3$, then $\{1,2\}$ is an index set of $p$ in $\pi$ making the occurrence graph $G_{p}(\pi)$ non-empty, see Figure 6.9.

If $\pi^{\prime}(2) \leq n-3$ then $\pi^{\prime}(2)(n+1), \pi^{\prime}(2)(n-1)$ and $\pi^{\prime}(2)(n-2)$ are all 


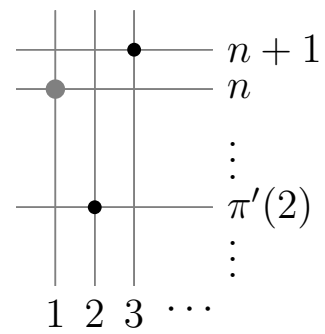

Figure 6.9: $k=n$ and $z=3$

occcurrences of $p$ in $\pi^{\prime}$, resulting in $G_{p}\left(\pi^{\prime}\right)$ having a triangle.

If $\pi^{\prime}(2)=n-1$ then $\{1,3\}$ and $\{2,3\}$ is an isolated path component in $G_{p}\left(\pi^{\prime}\right)$ and $\pi^{\prime}=n(n-1)(n+1)(n-2) \cdots 1$ is the only permutation such that the occurrence graph is a tree.

We therefore assume $\pi^{\prime}(2)=n-2$, see Figure 6.10 .

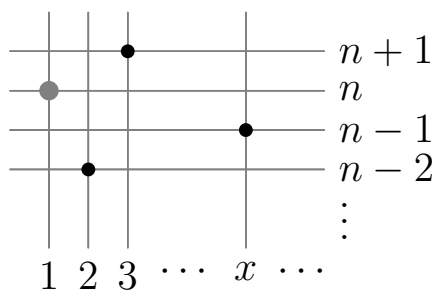

Figure 6.10: $k=n, z=3$ and $\pi^{\prime}(2)=n-2$

Let $\sigma=\operatorname{st}\left(\pi^{\prime}(2) \cdots \pi^{\prime}(n+1)\right)$. Note that the occurrence graphs $G_{p}\left(\pi^{\prime}\right)$ and $G_{p}(\sigma)$ are the same except the former has an the extra vertex $\{1,2\}$ and an edge connecting it to a graph corresponding to $G_{p}(\sigma)$. Therefore, $G_{p}\left(\pi^{\prime}\right)$ is a tree if and only if $G_{p}(\sigma)$ is a tree.

Note that $\sigma(1)=n-2$ and $\sigma(2)=n$ and therefore $\sigma$ is like $\pi^{\prime}$ in the case $k=n-1$ and $z=2$ as in Figure 6.7, only of length $n$ instead of $n+1$. By the same reasoning as in that case the number of permutations $\sigma$ (and therefore $\pi^{\prime}$ ) such that $G_{p}\left(\pi^{\prime}\right)$ is a tree is $T(n)=n-2$.

iii) If $z=2$, then $\{1,2\}$ is an isolated vertex in $G_{p}\left(\pi^{\prime}\right)$, see Figure 6.11. The occurrence graph of $p$ in $\pi^{\prime}$ is a tree if and only if $G_{p}(\pi)$ is the empty graph which is true if and only if $\pi$ is the decreasing permutation. Therefore there is only one permutation $\pi^{\prime}=n(n+1)(n-1) \ldots 1$ such that $G_{p}\left(\pi^{\prime}\right)$ is a tree. 


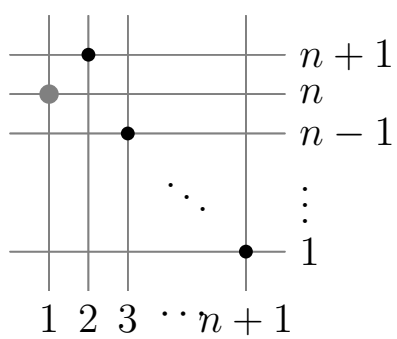

Figure 6.11: $k=n$ and $z=2$

To sum up the the case $k=n$ there are $1+(n-2)+1=n$ permutation $\pi^{\prime}$ such that $G_{p}\left(\pi^{\prime}\right)$ is a tree.

(IV) $k=n+1$ : Every occurrence $\pi(i) \pi(j)$ of $p$ in $\pi$ is also an occurrence of $p$ in $\pi^{\prime}$, but with index set $\{i+1, j+1\}$ instead of $\{i, j\}$. There are no more occurrences of $p$ in $\pi^{\prime}$ because $\pi^{\prime}(1)=n+1>\pi^{\prime}\left(j^{\prime}\right)$ for every $j^{\prime}>1$ so $\pi^{\prime}(1) \pi^{\prime}\left(j^{\prime}\right)$ is not an occurrence of $p$ for any $j^{\prime}>1$.

This means that $G_{12}\left(\pi^{\prime}\right) \cong G_{12}(\pi)$ so by the induction hypothesis we obtain that there are $(n-1)^{2}$ permutations $\pi^{\prime}$ such that the occurrence graph is a tree for this value of $k$.

To sum up the four instances there is a total of $0+(n-1)+n+(n-1)^{2}=n^{2}$ permutations $\pi^{\prime}$ such that $G_{p}\left(\pi^{\prime}\right)$ is a tree. 



\section{Future work}

There are some open questions about occurrence graphs that would be interesting to look into. Is the function $G_{p}(\pi)$ continuous is some sense? Can we make $G_{p}$ into a functor? For permutations $p, q, \pi, \sigma$, how do the skew sum and direct sum of the permutations $\pi$ and $\sigma$ affect the occurrence graphs $G_{p}(\pi), G_{p}(\sigma), G_{p}(\pi \oplus \sigma)$, $G_{p}(\pi \ominus \sigma)$ ? What about $G_{p \oplus q}(\pi)$ and $G_{p \ominus q}(\pi)$ ? 



\section{Bibliography}

[1] A. Claesson, B. E. Tenner and H. Ulfarsson, Coincidence among families of mesh patterns, submitted. arXiv:1412.0703

[2] D. Callan, Pattern avoidance in "flattened" partitions, Discrete Math. 309 (2009) 4187-4191

[3] S. L. Vidarsdottir and H. Ulfarsson, Isomorphisms between consecutive pattern classes, http://hdl .handle.net/1946/20561

[4] W. A. Stein et al. Sage Mathematics Software (Version 6.5), The Sage Development Team, 2015, http://www.sagemath.org 